\title{
Ten Years of the Collaborative Cross
}

\author{
David W. Threadgill*,1 and Gary A. Churchill ${ }^{\dagger}$ \\ *Department of Genetics, North Carolina State University, Raleigh, North Carolina 27695, and ${ }^{\dagger}$ The Jackson Laboratory, Bar \\ Harbor, Maine 04609
}

$T^{\text {H }}$ HE February 2012 issues of GENETICS and G3: GENES, GENOMES, GENETICS present a collection of articles reporting recent advances from the international Collaborative Cross (CC) project. The goal of the CC project is to develop a new resource that will enhance quantitative trait locus (QTL) and systems genetic analyses in mice. The CC consists of hundreds of independently bred, octo-parental recombinant inbred lines (Figure 1). The work reported in these issues represents progress toward completion of the CC, proof-of-principle experiments utilizing incipient inbred CC mice, and new research areas and complementary resources facilitated by the CC project.

\section{Collaborative Cross Past}

The idea to develop a multi-parental recombinant inbred panel was conceived $>10$ years ago in response to a challenge positing that discovering the genetic basis of complex traits would be more efficient using genome-wide mutagenesis (Nadeau and Frankel 2000), which at the time was undergoing a renaissance. Subsequently, the concept for a new, purpose-built mouse resource was presented at a satellite meeting of the 15th International Mouse Genome Conference in Edinburgh, Scotland (Threadgill et al. 2002). The Complex Trait Consortium (CTC; since renamed Complex Trait Community) was also established during the satellite meeting.

A committee from the CTC met the following year to debate the structure and specifics of a breeding design that would mix and randomize multiple genomes into an experimental population where each recombination site was unique and whose size was sufficiently powered to support QTL analysis at near single-gene resolution (supporting information, File S1). Because of community involvement in designing and implementing this new resource, it became

Copyright (C) 2012 by the Genetics Society of America doi: 10.1534/genetics.111.138032

Supporting information is available online at http://www.genetics.org/lookup/suppl/ doi:10.1534/genetics.111.138032/-/DC1.

${ }^{1}$ Corresponding author: Department of Genetics, Thomas Hall 3510 CB\#7614, North

Carolina State University, Raleigh, NC 27695. E-mail: threadgill@ncsu.edu known as the Collaborative Cross (Churchill et al. 2004). Subsequent discussions with the broader genetics community led to the realization that the CC would be far more than a new QTL mapping resource, but a resource that could support integrative, systems-level biology or "systems genetics" in mice (Threadgill 2006).

After extensive discussions to determine which extant inbred strains (the "founders") would be used as the initiating germplasm, eight strains were selected from existing laboratory and wild-derived strains representing the three major Mus musculus subspecies (M. m. musculus, M. m. domesticus, and M. m. castaneous): A/J, C57BL/6J, 129Sv/ ImJ, NOD/LtJ, NZO/H1J, CAST/EiJ, PWK/PhJ, and WSB/ EiJ. Genetic analysis revealed that the eight strains captured almost $90 \%$ of the known genetic variation present in laboratory mice originating from $M$. musculus and that the captured variation is randomly distributed across the genome (Roberts et al. 2007).

The CC project was formally initiated in 2004 at The Jackson Laboratory through the generation of a full, reciprocal diallel cross among the eight founder strains. The resulting $\mathrm{F}_{1}$ population was shipped to three breeding sites-Oak Ridge, Tennessee (Oak Ridge National Laboratory); Perth, Australia (Western Australia Institute for Medical Research/Geniad Ltd.); and Nairobi, Kenya (International Livestock Research Institute)_for two subsequent crosses to generate octo-parental mice from which inbred CC lines were developed. The U.S. population was eventually moved to Chapel Hill, North Carolina (University of North Carolina) and the Kenyan population to Tel Aviv, Israel (Tel Aviv University). Interim reports from the three major breeding centers were previously published (Chesler et al. 2008; Iraqi et al. 2008; Morahan et al. 2008).

During the 10-year journey to develop the CC, many additional research areas were facilitated, including analysis and simulation of expected numbers of recombination sites and the rate of inbreeding in multi-parental inbred lines (Broman 2005) and QTL mapping simulations to predict the eventual power of the CC (Valdar et al. 2006). Unanticipated challenges, such as the observation that the majority of initiated CC lines become extinct, were also encountered. 

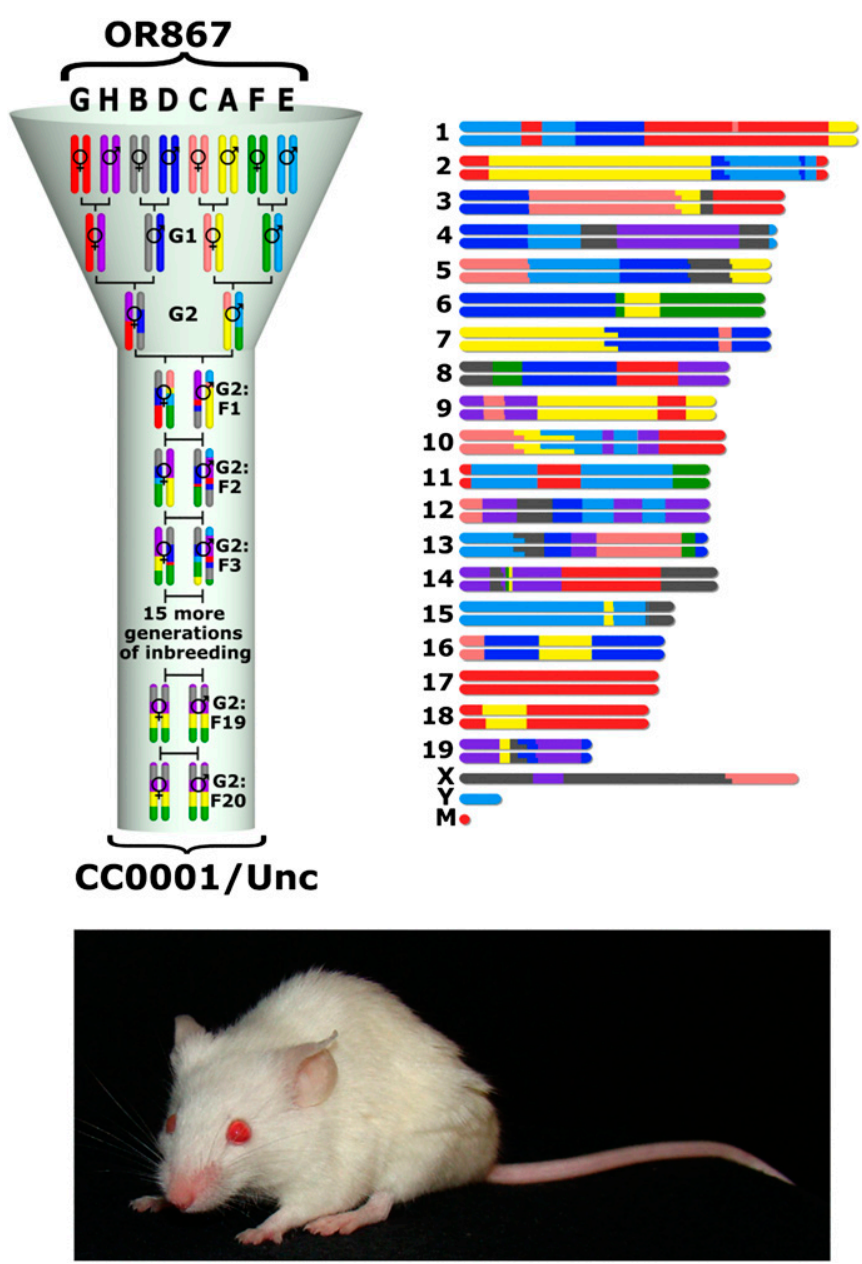

Figure 1 Development of an individual CC line. Each CC line originates from an independently breeding funnel so that every recombination site in the CC population is uniquely generated. In this example, a CC line is produced from the breeding line with laboratory code OR867. The first CC line to be declared inbred was from this breeding funnel and was given the official designation "CC0001/Unc." A representation of the genome color coded by founder strain contribution is shown next to the breeding funnel, and a photograph of a mouse from CC0001/Unc is shown below.

\section{Collaborative Cross Present}

Initial genetic and phenotypic analyses of the CC revealed the absence of large-scale selection within the CC population; balanced founder allele frequencies; dense, evenly distributed recombination sites; and phenotype distributions exceeding other extant resources (Aylor et al. 2011; Philip et al. 2011). Mapping resolution of expression QTL (eQTL) was shown to be $<1 \mathrm{Mb}$ using as few as 156 mice from incipient inbred CC lines (called pre-CC mice). Analysis of the CC genome architecture has been greatly extended in an article from the Collaborative Cross Consortium in this issue of GENETICS (Collaborative Cross Consortium 2012). The authors report a detailed genetic analysis of extant CC lines from the three breeding populations. This analysis revealed a small number of lines with breeding errors, such as missing founders, but also confirmed previous analysis showing that the founder haplotypes are inherited at expected frequencies. Evidence of transmission ratio distortion shared in all three independently breeding CC populations is also presented, suggesting a biological basis rather than a husbandry cause. The three breeding sites used different husbandry practices ranging from computer-assisted randomization of brother-sister matings to harem sibling matings and withinlineage backcrossing. The most important finding from this work was the absence of gametic disequilibrium in the CC population. Interchromosomal associations are extensive in other inbred strains, which is the underlying cause for high false-positive rates in genome-wide association studies when using the existing populations of mouse strains (Payseur and Place 2007).

Monitoring the inbreeding rates within CC lines confirmed that an extended number of generations would be needed to reach inbred status for many of the CC lines (Broman 2005). An article in G3 by Welsh and McMillan reports the development of a genotype-driven, accelerated inbreeding technique that is being exploited to overcome the lengthy process needed to achieve fixation of regions with heterozygosity (2012). Simulations demonstrated that the number of generations to reach inbred status using randomized sib-mating can be substantially reduced with minimal impact on the number of retained recombination sites by using a combination of parental backcrossing and markerassisted inbreeding. The number of generations can be reduced by almost 10, while reducing the number of recombination sites in the final line by $<5 \%$. The marker-assisted inbreeding approach is currently being used to complete the inbreeding of CC lines from all three breeding sites. Until all CC lines are inbred, many investigators are using pre-CC mice from intermediate generations in proof-of-principle experiments. For example, previous studies have mapped and proposed candidate genes for susceptibility to Aspergillus fumigatus infection and energy balance using pre-CC mice (Durrant et al. 2011; Mathes et al. 2011).

The power and resolution of mapping studies using preCC mice is further demonstrated in articles in G3 by Kelada et al. (2012) and Bottomly et al. (2012). Kelada et al. (2012) elucidated genetic factors controlling hematological parameters including red blood cell volume, white blood cell count, percentage of neutrophils, and monocyte number. They proposed a number of candidate genes by narrowing QTL intervals in the context of phylogeny. Similarly, Bottomly et al. (2012) used pre-CC mice to elucidate the genetic control of eQTL in mice with extreme response to influenza A infection. These studies employ regression analysis on eight founder haplotypes (Aylor et al. 2011). The pattern of allele effects across the eight haplotypes used in conjunction with whole-genome sequences provides a greatly reduced set of candidate SNPs in contrast to two-way crosses where all segregating SNPs are candidate causal loci.

Analysis methods for pre-CC mice remain an area of rapid development. In an article by Broman in this issue of GENETICS, a new approach using $k$-step probabilities for 
a Markov chain on genotype states is presented to infer genotype probabilities at intermediate stages of inbreeding (Broman 2012a). In addition to the elegant mathematics used in their derivation, these probabilities have utility for analyses of pre-CC populations. Computational methods presented in an article by Z. Zhang et al. in G3 also improve the analysis of pre-CC data (2012). Zhang et al. present a new association mapping technique using semiperfect phylogenetic trees that make allowance for heterozygous regions found in pre-CC mice. They validate the analysis using the Mendelian trait "white spotted blaze" that segregates in the CC population from the WSB/EiJ founder and the quantitative phenotype running distance.

The Diversity Outbred (DO), a complementary resource to the CC, was initiated at The Jackson Laboratory using CC lines from the U.S. population at intermediate stages of inbreeding. An article in this issue of GENETICS by Svenson et al. provides the first description and genetic analysis using the DO population (2012). The DO is a population of mice with the same genetic composition as the CC but which is maintained outbred through random matings, which increases the mapping resolution through accumulation of additional recombination sites. Methods for analyzing the multi-allelic outbred DO population are presented and validated using the identification of Foxo1 as a candidate for a QTL-regulating serum cholesterol. A second analytical paper by Broman in G3 (2012b) provides key results for computing genotype probabilities in DO mice. An analysis of a commercial outbred stock, presented in a G3 article by W. Zhang et al., underscores the lack of genetic diversity in current mouse stocks that motivated the creation of the CC and DO populations (2012). Data presented in these articles confirm and extend the power of a previous heterogeneous stock also developed from the CC founders (Iancu et al. 2010).

Numerous other advances in complex trait analysis have been facilitated by the CC project. An article by Wang et al. in this issue of GENETICS describes a new method to impute SNPs using local phylogeny trees (2012) and whole-genome sequences from 17 inbred strains, including the eight CC founders (Keane et al. 2011). Imputed genomes were generated for 100 extant mouse strains resulting in almost 1 billion new genotype calls with $<0.4 \%$ error genome-wide. This work greatly extends previous work to use genome imputation for genome ancestry in complex inbreeding pedigrees (Liu et al. 2010) and will support genetic and genomic analyses of extant inbred strains whose genomes have not been sequenced.

Another advance reported in this issue of GENETICS is an improvement in the analysis of diallel crosses, which have been previously used to characterize the aggregate effects of genetic background on phenotypes. The article by Lenarcic et al. analyzes phenotypic data obtained from mice from the diallel of founders used to initiate production of the CC. They employ a new Bayesian method for analysis of diallel crosses that improves decomposition of phenotypic variance into biologically intuitive components (Lenarcic et al. 2012).

\section{Collaborative Cross Future}

The CC will become the primary platform for systems-level analysis in mammals. Using the brain as a model multicompartment organ, Sun et al. report in an article in G3 that interstrain variation in gene expression is greater in discrete brain regions compared to interstrain transcriptional differences detected comparing whole brains (2012). This study has important implications for future whole-body dissections of CC mice for integrated systems analysis. The CC will continue to be an important stimulus for supporting QTL and systems genetics research. The CC has already proven to be a superior population for selective breeding (Zombeck et al. 2011) and will likely be exploited for future genetic selection experiments targeting specific phenotypes.

Future applications of the CC will exploit the strategy of recombinant inbred intercrosses (RIX), which are diallel crosses using panels of recombinant inbred lines (Zou et al. 2005), to substantially extend the numbers of unique genotypes. Compared to inbred lines, RIX have statistically lower phenotypic variances. In addition, RIX populations support analysis of parent-of-origin and transgenerational effects, which will further our understanding of genetic causes of phenotypes with complex etiologies. An article by Gong and Zou in this issue of GENETICS builds upon previous RIX studies by presenting a flexible, nonparametric time-varying coefficient QTL mapping method for RIX data (2012). This work will support future use of the CC to investigate gene-by-time interactions as well as the genetic control of phenotypic variance as opposed to the classical analysis of phenotypic means (Ronnegard and Valdar 2011). Understanding genetic control of phenotypic variance is essential for improving the accuracy of predictive medicine.

Generation of RIX will further support investigations into the genetic architecture of extinct CC lines aimed at elucidating the causes of genome incompatibilities, which has important implications for reproductive medicine. The extinction that occurred in many individual CC lines could be due to fixation of incompatible alleles at different loci, presumably originating from the three $M$. musculus subspecies. Identifying homozygous allele combinations in extinct lines that are not observed in the extant CC lines will provide candidates that can be synthetically tested by recreating unique allele combinations using specific RIX from CC lines.

More than a decade after the conception of the Collaborative Cross, the first fully inbred strains are becoming available for researchers to use. The lead article in this issue of GENETICS presents a model for community access to the CC resource (Collaborative Cross Consortium 2012). Maintenance and distribution of the new lines will present challenges that require ongoing community support. If the CC is to deliver on its promise, it must be used. In-depth characterization of phenotypes spanning multiple domains of mammalian biology will be needed to develop a systemslevel perspective. New databases and analytical tools are already under development (http://compgen.unc.edu/wp/ 
and http://churchill.jax.org/research/cgd.shtml). Experimental designs that combine the CC, DO, and other resources, such as the comprehensive mouse knockout panel, are being proposed to extend the scope of investigations using the CC strains. The energy and ingenuity of the community of users who have been awaiting the arrival of this resource is ready to be unleashed. The articles in the current issues of GENETICS and G3 provide just a glimpse of what is to come.

\section{Literature Cited}

Aylor, D. L., W. Valdar, W. Foulds-Mathes, R. J. Buus, R. A. Verdugo et al., 2011 Genetic analysis of complex traits in the emerging Collaborative Cross. Genome Res. 21: 1213-1222.

Bottomly D., M. T. Ferris, L. D. Aicher, E. Rosenzweig, A. Whitmore et al., 2012 Expression quantitative trait loci for extreme host response to influenza A in pre-Collaborative Cross mice. G3: Genes, Genomes, Genetics 2: @@@-@@@.

Broman, K. W., 2005 The genomes of recombinant inbred lines. Genetics 169: 1133-1146.

Broman, K. W., 2012a Genotype probabilities at intermediate generations in the construction of recombinant inbred lines. Genetics 190: @@@-@@@.

Broman, K. W., 2012b Haplotype probabilities in advanced intercross populations, G3: Genes, Genomes, Genetics 2: @@@-@@@.

Chesler, E. J., D. R. Miller, L. R. Branstetter, L. D. Galloway, B. L. Jackson et al., 2008 The Collaborative Cross at Oak Ridge National Laboratory: developing a powerful resource for systems genetics. Mamm. Genome 19: 382-389.

Churchill, G. A., D. C. Airey, H. Allayee, J. M. Angel, A. D. Attie et al., 2004 The Collaborative Cross, a community resource for the genetic analysis of complex traits. Nat. Genet. 36: 1133-1137.

Collaborative Cross Consortium, 2012 The genome architecture of the Collaborative Cross mouse genetic reference population. Genetics 190:@@@-@@@.

Durrant, C., H. Tayem, B. Yalcin, J. Cleak, L. Goodstadt et al., 2011 Collaborative Cross mice and their power to map host susceptibility to Aspergillus fumigatus infection. Genome Res. 21: 1239-1248.

Gong, Y., and F. Zou, 2012 Varying coefficient models for mapping quantitative trait loci using recombinant inbred intercrosses. Genetics 190: @@@-@@@.

Iancu, O. D., P. Darakjian, N. A. Walter, B. Malmanger, D. Oberbeck et al., 2010 Genetic diversity and striatal gene networks: focus on the heterogeneous stock-collaborative cross (HS-CC) mouse. BMC Genomics 11: 585.

Iraqi, F. A., G. Churchill, and R. Mott, 2008 The Collaborative Cross, developing a resource for mammalian systems genetics: a status report of the Wellcome Trust cohort. Mamm. Genome 19: $379-381$.

Keane, T. M., L. Goodstadt, P. Danecek, M. A. White, K. Wong et al., 2011 Mouse genomic variation and its effect on phenotypes and gene regulation. Nature 477: 289-294.

Kelada, S. N. P., D. L. Aylor, B. C. E. Peck, J. F. Ryan, U. Tavarez et al., 2012 Genetic analysis of hematological parameters in incipient lines of the Collaborative Cross. G3: Genes, Genomes, Genetics 2: @@@-@@@.

Lenarcic, A. B., K. L. Svenson, G. A. Churchill, and W. Valdar, 2012 A general Bayesian approach to analyzing diallel crosses of inbred strains. Genetics 190: @@@-@@@.
Liu, E. Y., Q. Zhang, L. McMillan, F. P. De Villena, and W. Wang, 2010 Efficient genome ancestry inference in complex pedigrees with inbreeding. Bioinformatics 26: i199-i207.

Mathes, W. F., D. L. Aylor, D. R. Miller, G. A. Churchill, E. J. Chesler et al., 2011 Architecture of energy balance traits in emerging lines of the Collaborative Cross. Am. J. Physiol. Endocrinol. Metab. 300: E1124-E1134.

Morahan, G., L. Balmer, and D. Monley, 2008 Establishment of "The Gene Mine": a resource for rapid identification of complex trait genes. Mamm. Genome 19: 390-393.

Nadeau, J. H., and W. N. Frankel, 2000 The roads from phenotypic variation to gene discovery: mutagenesis $v s$. QTLs. Nat. Genet. 25: 381-384.

Payseur, B. A., and M. Place, 2007 Prospects for association mapping in classical inbred mouse strains. Genetics 175: 1999-2008.

Philip, V. M., G. Sokoloff, C. L. Ackert-Bicknell, M. Striz, L. Branstetter et al., 2011 Genetic analysis in the Collaborative Cross breeding population. Genome Res. 21: 1223-1238.

Roberts, A., F. Pardo-Manuel De Villena, W. Wang, L. McMillan, and D. W. Threadgill, 2007 The polymorphism architecture of mouse genetic resources elucidated using genome-wide resequencing data: implications for QTL discovery and systems genetics. Mamm. Genome 18: 473-481.

Ronnegard, L., and W. Valdar, 2011 Detecting major genetic loci controlling phenotypic variability in experimental crosses. Genetics 188: 435-447.

Sun, W., S. Lee, V. Zhabotynsky, F. Zou, F. A. Wright et al., 2012 Transcriptome atlases of mouse brain reveals differential expression across brain regions and genetic backgrounds. G3: Genes, Genomes, Genetics 2: @@@-@@@.

Svenson, K. L., D. M. Gatti, W. Valdar, C. E. Welsh, R. Cheng et al., 2012 High resolution genetic mapping using the Mouse Diversity Outbred Population. Genetics 190: @@@-@@@.

Threadgill, D. W., 2006 Meeting report for the 4th annual Complex Trait Consortium meeting: from QTLs to systems genetics. Mamm. Genome 17: 2-4.

Threadgill, D. W., K. W. Hunter, and R. W. Williams, 2002 Genetic dissection of complex and quantitative traits: from fantasy to reality via a community effort. Mamm. Genome 13: 175-178.

Valdar, W., J. Flint, and R. Mott, 2006 Simulating the collaborative cross: power of quantitative trait loci detection and mapping resolution in large sets of recombinant inbred strains of mice. Genetics 172: 1783-1797.

Wang, J. R., F. Pardo-Manuel de Villena, H. A. Lawson, J. M. Cheverud, G. A. Churchill et al., 2012 Imputation of single-nucleotide polymorphisms in inbred mice using local phylogeny. Genetics 190: @@@-@@@.

Welsh, C. E., and L. McMillan, 2012 Accelerating the inbreeding of multi-parental recombinant inbred lines generated by sibling matings. G3: Genes, Genomes, Genetics 2: @@@-@@@.

Zhang, W., R. Korstanje, J. Thaisz, F. Staedtler, N. Harttman et al., 2012 Genome-wide association mapping of quantitative traits in outbred mice. G3: Genes, Genomes, Genetics 2: @@@-@@@.

Zhang, Z., X. Zhang, and W. Wang, 2012 HTreeQA: using semiperfect phylogeny trees in quantitative trait loci study on genotype data. G3: Genes, Genomes, Genetics 2: @@@-@@@.

Zombeck, J. A., E. K. Deyoung, W. J. Brzezinska, and J. S. Rhodes, 2011 Selective breeding for increased home cage physical activity in collaborative cross and Hsd:ICR mice. Behav. Genet. 41: 571-582.

Zou, F., J. A. Gelfond, D. C. Airey, L. Lu, K. F. Manly et al., 2005 Quantitative trait locus analysis using recombinant inbred intercrosses: theoretical and empirical considerations. Genetics 170: 1299-1311. 


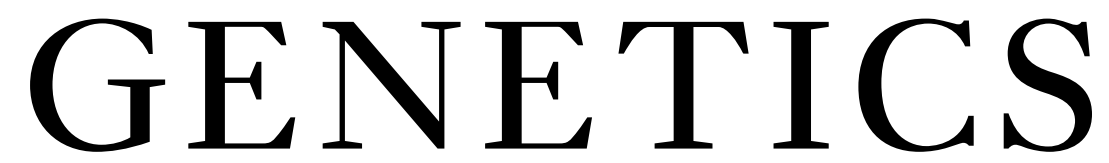

Supporting Information http://www.genetics.org/lookup/suppl/doi:10.1534/genetics.111.138032/-/DC1

\section{Ten Years of the Collaborative Cross}

David W. Threadgill and Gary A. Churchill 
File S1

Complex Trait Consortium - $1^{\text {st }}$ Workshop Report: September 2002

File S1 is available for download at http://www.genetics.org/lookup/suppl/doi:10.1534/genetics.111.138032/-/DC1. 